\title{
Hybrid Internal Models for Robust Spline Tracking
}

\author{
N. Cox, L. Marconi and A. R. Teel
}

\begin{abstract}
In this paper we consider the problem of tracking reference signals modeled by splines on the output of an uncertain linear system. The problem is cast in the hybrid output regulation setting. We show that any spline-based signal can be generated as the output of a linear hybrid exosystem that experiences jumps regularly over time. Then, we design a hybrid internal model-based regulator able to guarantee asymptotic tracking robustly with respect to parametric uncertainties in the plant. We show how the hybrid stabilization task implicitly present in the problem of output regulation hides interesting problems due to the fact that the hybrid exosystem generating the spline is neither continuous-time observable during flows nor discrete-time observable during jumps. Simulation results are provided in order to show the effectiveness of the design procedure.
\end{abstract}

\section{INTRODUCTION}

The problem of output regulation for hybrid linear systems has been recently investigated in [9] (see also [10]) by extending well-known results characterizing the theory in the continuous-time linear [3], [5] and nonlinear settings [8]. The proposed framework considers the class of linear systems and exosystems that are subject to jumps according to a known clock that satisfies a dwell-time constraint. For this class of systems design techniques based on the notion of a hybrid internal model have been developed and shown to be necessary for robust regulation. Further developments of the work [9] have been presented in [2], where the clock variable is unknown, and in [1], where linear systems with arbitrary relative degree are dealt with.

In this work we aim to add a further piece to the developed theory by considering the case in which the hybrid exosystem generates signals obtained by spline interpolation [11]. Spline interpolation is widely adopted in the robotic literature ([12]) in order to generate reference signals that smoothly interpolate waypoints by avoiding Runge's phenomenon, which usually appears while using polynomial interpolation. Splines have been shown to be effective in path generation of mobile robots [7], in the aerospace domain [13], and in many other applicative fields where efficient trajectory planning is a key requirement. Furthermore, they have been shown to be efficiently computable when dealing with actuation constraints in an optimal manner, see [4].

We build upon the general theory of [9] and design robust regulators for tracking cyclic signals obtained by

Nicholas Cox and A.R. Teel are with ECE Dept., University of California, Santa Barbara, USA njcox@umail.ucsb.edu, and teeldece.ucsb.edu. Research supported by AFOSR FA9550-091-0203, NSF ECCS-0925637

Lorenzo Marconi is with C.A.SY. - DEIS, University of Bologna, Bologna, Italy lorenzo.marconi@unibo.it. Research supported in part by the European Project AIRobots (ICT 248669). spline interpolation through the use of a hybrid internal model. The design problem at hand is interesting because the so-called steady state generator is neither continuoustime observable through the flow dynamics, nor discrete-time observable during jump relations. For illustrative reasons we focus on the class of continuous-time linear systems that are minimum-phase and relative degree one.

Notation For $x \in \mathbb{R},\lfloor x\rfloor$ denotes the largest integer such that $\lfloor x\rfloor \leq x$. For a square matrix, $M$, we say that $M \in \mathcal{D}_{1}$ if all the eigenvalues of $M$ lie strictly within the unitary disk. This work uses the framework, results and notation regarding hybrid systems presented as a tutorial in [6].

\section{GENERATING SPLINES THROUGH HYBRID LINEAR EXOSYSTEMS}

In this paper we focus on cyclic time reference signals $y^{\star}(t)$ obtained by periodically concatenating the basic signal, $\mathcal{B}(t)$, with a period given by $T$, where

$$
y^{\star}(t)=\mathcal{B}(t-i T), \quad i=\left\lfloor\frac{t}{T}\right\rfloor, t \geq 0, T>0,
$$

with $\mathcal{B}:[0, T] \rightarrow \mathbb{R}$ a sufficiently smooth function. The basic signal $\mathcal{B}(t)$ is thought of as being generated by using splines that pass through $N$ points $\left\{p_{1}, p_{2}, \ldots, p_{N}\right\}$ at time instances $\left\{t_{1}, t_{2}, \ldots, t_{N}\right\}$, namely $\mathcal{B}\left(t_{k}\right)=p_{k}, k=1, \ldots, N$. It is assumed that $t_{1}=0, t_{N}=T-T / N$ and $t_{k+1}-t_{k}=$ $T / N$ for all $k=1, \ldots, N-1$. We also assume that $\mathcal{B}(\cdot)$ is such that $\mathcal{B}(0)=\mathcal{B}(T)=p_{1}$ so that $y^{\star}: \mathbb{R}_{\geq 0} \rightarrow \mathbb{R}$ is a continuous function.

The idea behind spline generation ([11]) is to interpolate $N$ polynomials $\mathcal{P}_{k}(t):\left[t_{k}, t_{k+1}\right] \rightarrow \mathbb{R}$ of suitable order to guarantee that $\mathcal{P}_{k}\left(t_{k}\right)=p_{k}, \mathcal{P}_{k}\left(t_{k+1}\right)=p_{k+1}, k=$ $1, \ldots, N$ (with $t_{N+1}=T$ and $p_{N+1}=p_{1}$ ) and to smooth the time derivatives of the signal $y^{\star}(t)$ at the times $t_{k}$, $k=1, \ldots N+1$. For instance, by using polynomials of order three, simple algebraic arguments can be used to show that it is possible to design the four coefficients of the $N$ polynomials in such a way that the first and second time derivative (velocities and accelerations) of $y^{\star}(t)$ are continuous at $t=t_{k}, k=2, \ldots, N+1$. Smoother signals can be obtained by increasing the order of the polynomials $\mathcal{P}_{k}$. From now on, the polynomials $\mathcal{P}_{k}$ are assumed to be fixed in order to have continuity of $y^{\star}(t), \dot{y}^{\star}(t)$ and $\ddot{y}^{\star}(t)$ for all $t \geq 0$.

Next, we are interested in computing $y^{\star}(t)$ as the output 
of a hybrid linear exosystem of the form

$$
\left.\begin{array}{rl}
\dot{\tau} & =1 \\
\dot{w} & =S w
\end{array}\right\}(\tau, w) \in\left[0, \tau_{\max }\right] \times \mathbb{R}^{\nu},
$$

where $Q=\left[\begin{array}{ll}Q_{1} & 0_{1 \times N}\end{array}\right]$, the matrices $S$ and $J$ are to be defined, and $\tau_{\max }:=T / N$. The clock variable, $\tau$, determines the polynomial switching times. The construction of the exosystem is aided by breaking it into two sub-states, $w_{1} \in \mathbb{R}^{4}, w_{2} \in \mathbb{R}^{N}$, with the dimension of the sub-state $w_{1}$ dependent on the order of the polynomials that are used as the basic spline functions.

Let $S_{1} \in \mathbb{R}^{4} \times \mathbb{R}^{4}$ be the matrix whose elements are all zero except along the superdiagonal, which is filled with ones, and let $Q_{1}=\left(\begin{array}{cc}1 & 0_{1 \times 3}\end{array}\right)$. Furthermore, let

$$
\mathcal{Q}:=\left(\begin{array}{c}
Q_{1} \\
Q_{1} S_{1} \\
Q_{1} e^{S_{1} \tau_{\max }} \\
Q_{1} S_{1} e^{S_{1} \tau_{\max }}
\end{array}\right), \mathbf{v}_{k}:=\left(\begin{array}{c}
p_{k} \\
v_{k} \\
p_{k+1} \\
v_{k+1}
\end{array}\right), k=1, \ldots, N,
$$

with $v_{k}=\lim _{t \rightarrow t_{k}^{+}} \dot{\mathcal{P}}_{k}(t)$ and $v_{k+1}=\lim _{t \rightarrow t_{k+1}^{-}} \dot{\mathcal{P}}_{k}(t)$. The initial condition of the component $w_{1}$ of the exosystem is set to $w_{1}\left(t_{1}\right)=\mathcal{Q}^{-1} \mathbf{v}_{1}$, so that $y^{\star}(t)=Q_{1} w_{1}(t)$ for $t \in\left[t_{1}, t_{2}\right]$.

Then we have to identify the switching rule of the state of the exosystem at times $t_{k}, k=2, \ldots, N+1$ in order to reproduce the reference at times $t>t_{2}$. To this purpose, we observe that the value $w_{1}^{+}\left(t_{k+1}\right)$ needed to reproduce $\mathcal{P}_{k+1}(t)$ with $t \in\left[t_{k+1}, t_{k+2}\right], k=1, \ldots N-1$, is

$$
w_{1}^{+}\left(t_{k+1}\right)=\mathcal{Q}^{-1} \mathbf{v}_{k+1} \text {. }
$$

We observe that $p_{k+1}$ and $v_{k+1}$ can be expressed as a function of $w_{1}\left(t_{k+1}\right)$ since $p_{k+1}=Q_{1} w_{1}\left(t_{k+1}\right)$, and $v_{k+1}=Q_{1} S_{1} w_{1}\left(t_{k+1}\right)$. It is possible to express $v_{k+2}$ as function of $p_{k}, p_{k+1}, p_{k+2}, v_{k}$ and $v_{k+1}$, by imposing continuity in the acceleration at time $t_{k+1}$. In fact, by imposing $Q_{1} S_{1}^{2} w_{1}\left(t_{k+1}\right)=Q_{1} S_{1}^{2} w_{1}\left(t_{k+1}\right)^{+}$, and using

$$
w_{1}\left(t_{k+1}\right)=e^{S_{1} \tau_{\max }} w_{1}\left(t_{k}\right)^{+}=e^{S_{1} \tau_{\max }} \mathcal{Q}^{-1} \mathbf{v}_{k},
$$

one obtains

$$
Q_{1} S_{1}^{2} e^{S_{1} \tau_{\max }} \mathcal{Q}^{-1} \mathbf{v}_{k}=Q_{1} S_{1}^{2} \mathcal{Q}^{-1} \mathbf{v}_{k+1},
$$

which, solved for $v_{k+2}$, yields

$$
v_{k+2}=\Gamma\left(\begin{array}{ccccc}
p_{k} & p_{k+1} & p_{k+2} & v_{k} & v_{k+1}
\end{array}\right)^{T},
$$

with $\Gamma=\left(\begin{array}{lllll}-3 / \tau_{\max } & 0 & 3 / \tau_{\max } & -1 & -4\end{array}\right)$. By embedding the previous relation in (2) we obtain

$$
w_{1}^{+}\left(t_{k+1}\right)=L\left(\begin{array}{lllll}
p_{k} & p_{k+1} & p_{k+2} & v_{k} & v_{k+1}
\end{array}\right)^{T},
$$

where

$$
L=\left(\begin{array}{ccccc}
0 & 1 & 0 & 0 & 0 \\
0 & 0 & 0 & 0 & 1 \\
\frac{6}{\tau_{\max }^{2}} & \frac{-6}{\tau_{\max }^{2}} & 0 & \frac{2}{\tau_{\max }} & \frac{4}{\tau_{\max }} \\
\frac{-18}{\tau_{\max }^{3}} & \frac{12}{\tau_{\max }^{3}} & \frac{6}{\tau_{\max }^{3}} & \frac{-6}{\tau_{\max }^{2}} & \frac{-18}{\tau_{\max }^{2}}
\end{array}\right)
$$

Now, we observe that $p_{k}=Q_{1} e^{-S_{1} \tau_{\max }} w_{1}\left(t_{k+1}\right)$, $p_{k+1}=Q_{1} w_{1}\left(t_{k+1}\right), v_{k}=Q_{1} S_{1} e^{-S_{1} \tau_{\max }} w_{1}\left(t_{k+1}\right), v_{k+1}=$ $Q_{1} S_{1} w_{1}\left(t_{k+1}\right)$. To write a relation of the form $w^{+}\left(t_{k+1}\right)=$ $J w\left(t_{k}\right)$ we are thus left to express $p_{k+2}$ as function of the state of the exosystem. By preserving the linearity of the exosystem, this can be done by "enriching" the exosystem with additional states $w_{2} \in \mathbb{R}^{N}$ governed by the following dynamics (implementing a shift register)

$$
\begin{aligned}
\dot{w}_{2} & =0, \\
w_{2}^{+} & =J_{22} w_{2},
\end{aligned}
$$

where

$$
J_{22}=\left(\begin{array}{cc}
0_{1 \times N-1} & 1 \\
I_{N-1} & 0_{N-1 \times 1}
\end{array}\right)
$$

with the initial condition $w_{2}\left(t_{1}\right)=\left(\begin{array}{cc}1 & 0_{1 \times N-1}\end{array}\right)^{T}$. In this way $p_{k+2}=P w_{2}\left(t_{k+1}\right)$, with $P=$ $\left(\begin{array}{lllll}p_{3} & \cdots & p_{N} & p_{1} & p_{2}\end{array}\right)$, and

$w_{1}^{+}\left(t_{k+1}\right)=L\left(\begin{array}{cc}Q_{1} e^{-S_{1} \tau_{\max }} & 0 \\ Q_{1} & 0 \\ 0 & P \\ Q_{1} S_{1} e^{-S_{1} \tau_{\max }} & 0 \\ Q_{1} S_{1} & 0\end{array}\right)\left(\begin{array}{c}w_{1}\left(t_{k+1}\right) \\ w_{2}\left(t_{k+1}\right)\end{array}\right)$.

Overall, the exosystem, (1), takes the form

$$
\left.\begin{array}{rl}
\dot{\tau} & =1 \\
\dot{w}_{1} & =S_{1} w_{1} \\
\dot{w}_{2} & =0 \\
\tau^{+} & =0 \\
w_{1}^{+} & =J_{11} w_{1}+J_{12} w_{2} \\
w_{2}^{+} & =J_{22} w_{2}
\end{array}\right\}(\tau, w) \in\left[0, \tau_{\max }\right] \times \mathbb{R}^{\nu},
$$

with $y^{\star}=Q_{1} w_{1}$, where

$$
S_{1}=\left(\begin{array}{c}
0_{3 \times 1} I_{3} \\
0_{1 \times 4}
\end{array}\right), J_{11}=\left(\begin{array}{cc}
I_{3} & 0_{3 \times 1} \\
& L_{1}
\end{array}\right), J_{12}=\left(\begin{array}{c}
0_{3 \times N} \\
L_{2}
\end{array}\right),
$$

with $L_{1}$ and $L_{2}$ appropriately defined, and $J_{22}$ defined as in (4). Note that the matrices $S$ and $J$ are implicitly defined.

\section{THE ROBUST OUTPUT TRACKING PROBLEM AND THE REGULATOR DESIGN PRINCIPLES}

We are interested in designing controllers that force the output of a linear system to track a spline-generated reference signal of the form introduced in the previous section. For the sake of simplicity we limit our attention to regulated plants that are continuous-time and that have unitary relative degree. It must be observed, however, that all the forthcoming results can be extended in a quite straightforward way to deal with general hybrid linear systems with arbitrary relative degree. Without loss of generality, we thus focus on the systems modeled in the Brunowsky canonical form

$$
\begin{aligned}
& \dot{z}=A_{11} z+A_{12} y, \\
& \dot{y}=A_{21} z+A_{22} y+b u,
\end{aligned}
$$

in which $u \in \mathbb{R}$ and $y \in \mathbb{R}$ are, respectively, the control input and the regulated output, while $z \in \mathbb{R}^{n}$. All the matrices in (5) have appropriate dimensions, with the high frequency 
gain, $b \neq 0$ and assumed positive, without loss of generality. We associate a tracking error to the system, defined as

$$
e=y-y^{\star}=y-Q w,
$$

where $y^{\star}$ is the spline-based periodic signal introduced in Section II. Our goal is to find a hybrid regulator that only processes the error, $e$, and steers it, asymptotically, to zero despite possible parametric uncertainties in the plant, (5).

The problem at hand is a problem of hybrid linear output regulation (see [9]) in which hybrid internal model principles must be used to secure robust regulation. In this respect we follow the main design principles presented in [9].

We limit the analysis to minimum-phase systems. Namely, systems that fulfill the following assumptions.

Assumption 1 (minimum-phase) The matrix $A_{11}$ is such that $\exp \left(A_{11} \tau_{\text {max }}\right) \in \mathcal{D}_{1} \cdot \triangleleft$

Furthermore, in order to have $z$-dynamics with a welldefined steady state, we assume the following non-resonance condition between the zeros of system (5) and the "poles" of the hybrid exosystem, (1).

Assumption 2 (non-resonance condition) The following holds: $\operatorname{eig}\left(\exp \left(A_{11} \tau_{\max }\right)\right) \cap \operatorname{eig}\left(J \exp \left(S \tau_{\max }\right)\right)=\emptyset . \triangleleft$

With assumption 2 in hand, and with [9] in mind, we let $\Pi_{z}(\tau):\left[0, \tau_{\max }\right] \rightarrow \mathbb{R}^{n \times(4+N)}$ be the continuously differentiable function that is the unique solution of

$$
\begin{aligned}
\frac{d \Pi_{z}(\tau)}{d \tau} & =A_{11} \Pi_{z}(\tau)-\Pi_{z}(\tau) S+A_{12} Q, \\
0 & =\Pi_{z}\left(\tau_{\max }\right)-\Pi_{z}(0) J .
\end{aligned}
$$

Furthermore we consider the change of variables

$$
z \mapsto \tilde{z}=z-\Pi_{z}(\tau) w, \quad y \mapsto e=y-Q w,
$$

which transforms the system into a hybrid system flowing according to

$$
\begin{aligned}
& \dot{\tau}=1, \quad \dot{w}=S w, \\
& \dot{\tilde{z}}=A_{11} \tilde{z}+A_{12} e, \\
& \dot{e}=A_{21} \tilde{z}+A_{22} e+b(u-R(\tau) w),
\end{aligned}
$$

whenever $(\tau, w, \tilde{z}, e) \in\left[0, \tau_{\max }\right] \times \mathbb{R}^{4+N} \times \mathbb{R}^{n} \times \mathbb{R}$, where

$$
R(\tau)=\frac{1}{b}\left(Q S-A_{22} Q-A_{21} \Pi_{z}(\tau)\right),
$$

and jumping according to (using $Q_{1}=Q_{1} J_{11}$ and $Q_{1} J_{12}=0$ )

$$
\begin{aligned}
& \tau^{+}=0, w^{+}=J w, \\
& \tilde{z}^{+}=\tilde{z}, \tilde{e}^{+}=e,
\end{aligned}
$$

whenever $(\tau, w, \tilde{z}, e) \in\left\{\tau_{\max }\right\} \times \mathbb{R}^{4+N} \times \mathbb{R}^{n} \times \mathbb{R}$.

The goal of the regulator is to make the set $(\tilde{z}, e)=0$ globally exponentially stable for the error system, (6)-(8), by compensating for the term $R(\tau) w$. Note that $R(\tau) w$ is unknown since parametric uncertainties enter in the definition of $R(\tau)$. By following the prescriptions of [9] ([10]) we focus on a hybrid internal model-based regulator of the form

$$
\begin{aligned}
& \left.\begin{array}{l}
\dot{\tau}=1 \\
\dot{\eta}=F_{\mathrm{im}} \eta+G_{\mathrm{im}} u
\end{array}\right\}(\tau, \eta) \in\left[0, \tau_{\max }\right] \times \mathbb{R}^{\nu}, \\
& \left.\begin{array}{l}
\tau^{+}=0 \\
\eta^{+}=\Sigma_{\mathrm{im}} \eta
\end{array}\right\}(\tau, \eta) \in\left\{\tau_{\max }\right\} \times \mathbb{R}^{\nu}, \\
& u=\Gamma_{\mathrm{im}}(\tau) \eta+v \text {, }
\end{aligned}
$$

where $\nu \in \mathbb{N}, F_{\mathrm{im}}, G_{\mathrm{im}}, \Sigma_{\mathrm{im}}$ are matrices, $\Gamma_{\mathrm{im}}:\left[0, \tau_{\max }\right] \rightarrow$ $\mathbb{R}^{1 \times \nu}$ is a continuously differentiable function, and $v$ is a residual control input, all to be designed. The following result provides the main guidelines for the design of (9) as presented in [9].

Proposition 1: Let Assumptions 1 and 2 be fulfilled. Also, assume that the controller (9) is designed so that for some cont. diff. function $\Pi_{\eta}:\left[0, \tau_{\max }\right] \rightarrow \mathbb{R}^{\nu \times(4+N)}$ the set

$$
\mathcal{S}=\left\{(\tau, w, \eta) \in\left[0, \tau_{\max }\right] \times \mathbb{R}^{4+N} \times \mathbb{R}^{\nu}: \eta=\Pi_{\eta}(\tau) w\right\}
$$

is globally exponentially stable for the hybrid system

$$
\begin{aligned}
& \dot{\tau}=1, \quad \dot{w}=S w \quad\}(\tau, w, \eta) \\
& \left.\dot{\eta}=F_{\mathrm{im}} \eta+G_{\mathrm{im}} R(\tau) w\right\} \quad\left[0, \tau_{\max }\right] \times \mathbb{R}^{4+N} \times \mathbb{R}^{\nu}, \\
& \left.\tau^{+}=0, w^{+}=J w\right\}(\tau, w, \eta) \\
& \left.\eta^{+}=\Sigma_{\mathrm{im}} \eta \quad\right\} \in\left\{\tau_{\max }\right\} \times \mathbb{R}^{4+N} \times \mathbb{R}^{\nu},
\end{aligned}
$$

with

$$
\Gamma_{\mathrm{im}}(\tau) \Pi_{\eta}(\tau)=R(\tau) \quad \forall \tau \in\left[0, \tau_{\max }\right] .
$$

Then, there exists a $\kappa^{\star}>0$ such that for all $\kappa \geq \kappa^{\star}$ the regulator (9) with $v=-\kappa e$ solves the problem of hybrid output regulation. $\triangleleft$

As shown in [9] an internal model of the form (9) making the set (10) globally exponentially stable for (11) and fulfilling (12) always exists provided that the dimension $\nu$ is taken sufficiently large and the triplet $\left(F_{\mathrm{im}}, G_{\mathrm{im}}, \Sigma_{\mathrm{im}}\right)$ fulfills mild requirements. Indeed the following holds (see Proposition 4 in [10]).

Proposition 2: Let $\nu \geq N+4$ and $\left(F_{\mathrm{im}}, G_{\mathrm{im}}, \Sigma_{\mathrm{im}}\right)$ be chosen such that the pair $\left(F_{\mathrm{im}}, G_{\mathrm{im}}\right)$ is controllable, $\operatorname{eig}\left(\Sigma_{\mathrm{im}} \exp \left(F_{\mathrm{im}} \tau_{\text {max }}\right)\right) \cap \operatorname{eig}\left(J \exp \left(S \tau_{\text {max }}\right)\right)=\emptyset$, and $\Sigma_{\text {im }} \exp \left(F_{\text {im }} \tau_{\text {max }}\right) \in \mathcal{D}_{1}$. Then there exists a unique continuously differentiable function $\Pi_{\eta}:\left[0, \tau_{\max }\right] \rightarrow \mathbb{R}^{\nu \times(4+N)}$, that is the solution of

$$
\begin{aligned}
\frac{d \Pi_{\eta}(\tau)}{d \tau} & =F_{\mathrm{im}} \Pi_{\eta}(\tau)-\Pi_{\eta}(\tau) S+G_{\mathrm{im}} R(\tau), \\
0 & =\Sigma_{\mathrm{im}} \Pi_{\eta}\left(\tau_{\max }\right)-\Pi_{\eta}(0) J,
\end{aligned}
$$

such that the set $\mathcal{S}$ in (10) is globally exponentially stable for (11). Furthermore, the function $\Gamma_{\mathrm{im}}(\tau)=R(\tau) \Pi_{\eta}^{\dagger}(\tau)$, where $\Pi_{\eta}^{\dagger}(\tau)$ is the Moore-Penrose pseudo-inverse of $\Pi_{\eta}(\tau)$, is such that (12) is fulfilled. $\triangleleft$

The existence of a continuously differentiable function $\Pi_{\eta}(\tau)$ that is the solution of (13) follows from [9] by using the fact that $\Sigma_{\mathrm{im}} \exp \left(F_{\mathrm{im}} \tau_{\max }\right) \in \mathcal{D}_{1}$ and $\operatorname{eig}\left(\Sigma_{\text {im }} \exp \left(F_{\text {im }} \tau_{\text {max }}\right)\right) \cap \operatorname{eig}\left(J \exp \left(S \tau_{\max }\right)\right)=\emptyset$. While, the fact that $\nu \geq 4+N$ and that the pair $\left(F_{\mathrm{im}}, G_{\mathrm{im}}\right)$ is controllable plays a role in proving that $\Gamma_{\mathrm{im}}(\tau)=R(\tau) \Pi_{\eta}^{\dagger}(\tau)$ fulfills (12), as shown in Proposition 4 in [10].

Although the previous result shows that an error feedback regulator enforcing an asymptotically zero error can always be designed, it is not conclusive about the fact that such a regulator is continuously differentiable. As a matter of fact, there is no guarantee that $\Pi_{\eta}^{\dagger}(\tau)$, and thus $\Gamma_{\mathrm{im}}(\tau)$, is continuously differentiable. By following [14], it turns out that a sufficient condition under which the function $\Pi_{\eta}^{\dagger}(\tau)$, and thus $\Gamma_{\mathrm{im}}(\tau)$, is continuously differentiable, is the 
existence of an $r=\operatorname{rank}\left(\Pi_{\eta}(\tau)\right)$, such that $r \leq 4+N$ for all $\tau \in\left[0, \tau_{\max }\right]$. The fulfillment of such a sufficient condition is, in general, affected by all the matrices entering in (13), among which the pair $(S, J)$ define the hybrid exosystem.

Interestingly enough, simulation results show that in the case where the pair $(S, J)$ has the specific form presented in Section II for spline generation, the function $\Pi_{\eta}^{\dagger}(\tau)$ is not continuously differentiable for generic choices of the matrices $A_{11}, A_{12}, A_{21}$ and $A_{22}$ of the plant, (5). Namely, the $\operatorname{rank}\left(\Pi_{\eta}(\tau)\right)$ changes in the interval $\left[0, \tau_{\max }\right]$. Simulation results in Section VI give evidence of this fact. The design of a continuously differentiable internal model for the splinebased exosystem is thus more elaborate. A possible design is presented in the forthcoming section.

\section{A $\tau$-INDEPENDENT HYBRID INTERNAL MODEL}

For illustrative reasons, we consider the case in which the function $R(\tau)$ in (7) is $\tau$-independent (i.e. $\Pi_{z}(\tau) \equiv 0$ ). Due to the specific structure of $S$ and $Q, R$ can be partitioned as

$$
R=\left(\begin{array}{cc}
R_{1} & 0_{1 \times N}
\end{array}\right),
$$

with $R_{1}=\left(\begin{array}{llll}-A_{22} / b & 1 / b & 0 & 0\end{array}\right)$. Furthermore, note that the pair $\left(S_{1}, R_{1}\right)$ is observable.

This scenario is representative of cases in which the regulated plant does not have zero dynamics, or the steady state effect of the zero dynamics on the error dynamics has been compensated through feed forward control actions. We remark that even in this simplified setting the internal model prescribed by Proposition 2 is not continuously differentiable, and thus a more elaborate design is needed. It is worth stressing, however, that the design procedure herewith presented can be generalized to deal with generic, $\tau$-dependent, $R(\tau)$ functions.

By following Proposition 2, in the following part we design a quadruplet $\left(F_{\mathrm{im}}, G_{\mathrm{im}}, \Sigma_{\mathrm{im}}, \Gamma_{\mathrm{im}}\right)$ such that for some matrix $\Pi_{\eta} \in \mathbb{R}^{\nu \times(4+N)}$ the set $\mathcal{S}=\left\{(\tau, w, \eta) \in\left[0, \tau_{\max }\right] \times\right.$ $\left.\mathbb{R}^{4+N} \times \mathbb{R}^{\nu}: \eta=\Pi_{\eta} w\right\}$ is globally exponentially stable for the cascade of the system ${ }^{1}$

$$
\left.\begin{array}{c}
\dot{\tau}=1 \\
\dot{w}=S w \\
\tau^{+}=0 \\
w^{+}=J w \\
u_{\mathrm{ss}}=R w,
\end{array}\right\}(\tau, w) \in\left[0, \tau_{\max }\right] \times \mathbb{R}^{4+N},
$$

that feeds, through its output, $u_{\mathrm{ss}}$, the system

$$
\left.\begin{array}{l}
\dot{\tau}=1 \\
\dot{\eta}=F_{\mathrm{im}} \eta+G_{\mathrm{im}} u_{\mathrm{ss}}
\end{array}\right\}(\tau, \eta) \in\left[0, \tau_{\max }\right] \times \mathbb{R}^{\nu},
$$

and such that $\Gamma_{\mathrm{im}} \Pi_{\eta}=R$. The property required of system (16) is thus to be able to asymptotically reproduce, by its "output" $\Gamma_{\mathrm{im}} \eta$, all the output signals $u_{\mathrm{ss}}(t)=R w(t)$ generated by ${ }^{2}(15)$.

\footnotetext{
${ }^{1}$ System (15) is what, in the literature on output regulation, is usually referred to as a steady-state generator.

${ }^{2}$ This property is referred to as the (hybrid) internal model property in the literature on output regulation.
}

It is apparent that the problem of designing the quadruplet $\left(F_{\mathrm{im}}, G_{\mathrm{im}}, \Sigma_{\mathrm{im}}, \Gamma_{\mathrm{im}}\right)$ is very much related to the problem of observation of the state of (15) through the "output" $R w$. Indeed, by setting $\Gamma_{\mathrm{im}}=R$, the problem would be solved by designing (16) as a state observer of (15). This is the design strategy that is followed.

What makes the observation problem at hand interesting, per se, is that due to the specific structure of $S, J$ and $R$, the steady state generator is neither dynamically observable through the flow dynamics nor statically observable through the jump relation. Namely, the pairs $(S, R)$ and $(J, R)$ are not detectable. In particular, due to the structure of $S$ and $R$, it is apparent that the $w_{2}$ component is not detectable through the output, $R w$, of the continuous-time system $\dot{w}=S w$. Similarly, the structures of $J$ and $R$ imply that $(R w)^{+}=$ $R w$. Namely the state $w$, and thus, in particular, $w_{2}$, does not show up on the output during jumps.

Nevertheless, denoting by $w_{14}$ the fourth component of $w_{1}$ and noting that the jump rule governing $w_{14}$ is given by $w_{14}^{+}=L_{1} w_{1}+L_{2} w_{2}$, it turns out that $w_{2}$ shows up during jumps by affecting the value of $w_{14}$ that, in turn, is dynamically observable by the output, $R_{1} w_{1}$, of the flow dynamics $\dot{w}_{1}=S_{1} w_{1}$. The forthcoming proposition builds upon this intuition in order to design an observer of (15) using the intrinsic hybrid observability of the latter.

In order to make the previous intuition precise, we make the following assumption without loss of generality.

Assumption 3 The pair $\left(J_{22}, L_{2}\right)$ is observable. $\triangleleft$

Indeed, if $\left(J_{22}, R\right)$ were not observable ${ }^{3}$, after a canonical state transformation in the $w_{2}$ component, a reduced exosystem could be obtained by just replacing $\left(J_{22}, L_{2}\right)$ with its observable part, while preserving the ability of the resulting reduced-order steady state generator to generate all the output signals of the "full-order" system (15). The following proposition details a possible structure of (16).

Proposition 3: Let the pairs $\left(S_{1}, R_{1}\right)$ and $\left(J_{22}, L_{2}\right)$ be observable. Let system (16) be taken of the form

$$
\left.\begin{array}{rl}
\dot{\tau}= & 1 \\
\dot{\eta}_{1}= & S_{1} \eta_{1}+K_{1}\left(R_{1} \eta_{1}-u_{\mathrm{ss}}\right) \\
\dot{\eta}_{2}= & 0 \\
\left(\tau, \eta_{1}, \eta_{2}\right) & \in\left[0, \tau_{\max }\right] \times \mathbb{R}^{4} \times \mathbb{R}^{N}, \\
\tau^{+}= & 0 \\
\eta_{1}^{+}= & J_{11} \eta_{1}+J_{12}\left(\eta_{2}-K_{2} \eta_{14}\right) \\
\eta_{2}^{+}= & \left(J_{22}+K_{2} L_{2}\right)\left(\eta_{2}-K_{2} \eta_{14}\right) \\
& +K_{2} L_{1} \eta_{1} \\
\left(\tau, \eta_{1}, \eta_{2}\right) & \in\left\{\tau_{\max }\right\} \times \mathbb{R}^{4} \times \mathbb{R}^{N},
\end{array}\right\}
$$

where $\eta_{14}$ is the fourth component of $\eta_{1}$, with $K_{2}$ such that $\left(J_{22}+K_{2} L_{2}\right) \in \mathcal{D}_{1}$ and $K_{1}$ such that $\operatorname{Re}\left[\operatorname{eig}\left(S_{1}+K_{1} R_{1}\right)\right] \leq-g$, with $g$ a design parameter. Then there exists a $g>0$ such that the set $\mathcal{S}=\{(\tau, w, \eta) \in$ $\left.\left[0, \tau_{\max }\right] \times \mathbb{R}^{4+N} \times \mathbb{R}^{4+N}: \eta=\Pi_{\eta} w\right\}$, with

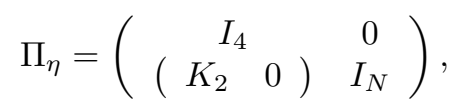

\footnotetext{
${ }^{3}$ In this respect it is interesting to observe that for some values of the waypoints $\left\{p_{1}, \ldots, p_{N}\right\}$, namely of the vector $P$ introduced in Section II, the pair in question might be unobservable.
} 
is globally exponentially stable for (15), (17). Moreover, if $\Gamma_{\mathrm{im}}=R$, then $\Gamma_{\mathrm{im}} \Pi_{\eta}=R$.

Proof: We change coordinates as $w_{2} \mapsto z=w_{2}+$ $K_{2} w_{14}, \eta_{1} \mapsto \tilde{\eta}_{1}=\eta_{1}-w_{1}$ and $\eta_{2} \mapsto \tilde{\eta}_{2}=\eta_{2}-z$. By using the fact that $\dot{w}_{14}=0$, systems (15) and (17), in the new coordinates, read as

$$
\begin{aligned}
& \left.\begin{array}{rl}
\dot{\tau} & =1 \\
\dot{w}_{1} & =S_{1} w_{1} \\
\dot{z} & =0
\end{array}\right\}\left(\tau, w_{1}, z\right) \in\left[0, \tau_{\max }\right] \times \mathbb{R}^{4} \times \mathbb{R}^{N} \\
& \tau^{+}=0 \\
& w_{1}^{+}=J_{11} w_{1}+J_{12}\left(z-K_{2} w_{14}\right) \\
& \left.z^{+}=\left(J_{22}+K_{2} L_{2}\right)\left(z-K_{2} w_{14}\right)+K_{2} L_{1} w_{1}\right\} \\
& \left(\tau, w_{1}, z\right) \in\left\{\tau_{\max }\right\} \times \mathbb{R}^{4} \times \mathbb{R}^{N},
\end{aligned}
$$

and

$$
\begin{aligned}
& \left.\begin{array}{rl}
\dot{\tau} & =1 \\
\dot{\tilde{\eta}}_{1} & =\left(S_{1}+K_{1} R_{1}\right) \tilde{\eta}_{1} \\
\dot{\tilde{\eta}}_{2} & =0
\end{array}\right\}\left(\tau, \tilde{\eta}_{1}, \tilde{\eta}_{2}\right) \in\left[0, \tau_{\max }\right] \times \mathbb{R}^{4} \times \mathbb{R}^{N}, \\
& \tau^{+}=0 \\
& \tilde{\eta}_{1}^{+}=J_{11} \tilde{\eta}_{1}+J_{12}\left(\tilde{\eta}_{2}-K_{2} \tilde{\eta}_{14}\right) \\
& \left.\tilde{\eta}_{2}^{+}=\left(J_{22}+K_{2} L_{2}\right) \tilde{\eta}_{2}-\left(J_{22}+K_{2} L_{2}\right) K_{2} \tilde{\eta}_{14}+K_{2} L_{1} \tilde{\eta}_{1}\right\} \\
& \left(\tau, \tilde{\eta}_{1}, \tilde{\eta}_{2}\right) \in\left\{\tau_{\max }\right\} \times \mathbb{R}^{4} \times \mathbb{R}^{N} .
\end{aligned}
$$

The latter subsystem can be compactly rewritten as $\dot{\tau}=1$, $\dot{\tilde{\eta}}=H_{e} \tilde{\eta}$ when $(\tau, \tilde{\eta}) \in\left[0, \tau_{\max }\right] \times \mathbb{R}^{4+N}$, and $\tau^{+}=0$, $\tilde{\eta}^{+}=J_{e} \tilde{\eta}$ when $(\tau, \tilde{\eta}) \in\left\{\tau_{\max }\right\} \times \mathbb{R}^{4+N}$, where $H_{e}$ and $J_{e}$ are properly defined. In particular, it turns out that if $K_{1}$ is chosen such that $\operatorname{Re}\left[\operatorname{eig}\left(S_{1}+K_{1} R_{1}\right)\right] \leq-g$ with $g$ sufficiently large, then $J_{e} \exp \left(H_{e} \tau_{\text {max }}\right) \in \mathcal{D}_{1}$. Hence, by standard results on sample data systems, $\left(\tilde{\eta}_{1}, \tilde{\eta}_{2}\right)$ converge to zero asymptotically by which the result follows.

In summary, the regulator (17), with $u=\Gamma_{\mathrm{im}} \eta+v$ replacing $u_{s s}$, satisfies the controller requirements for Proposition 1 and allows us to solve the hybrid output regulation problem for the spline exosystem.

\section{ROBUST HYBRID INTERNAL MODELS}

The internal model-based regulator obtained via the results of Propositions 2 and 3 is not robust because $\Gamma_{\text {im }}$ depends on $R$, where the latter is affected by possible parametric uncertainties of the plant. Here we sketch how a robust regulator can be obtained. As above, we limit the analysis to the case in which $R$ has the form (14) by remarking that the same ideas presented below, in principle, extend to the general case in which $R$ is a function of $\tau$ (see [9]).

The main intuition of the robust solution relies on the fact that any output signal that can be generated by the (uncertain) steady state generator (15) by varying the initial condition, $w(0)$, can also be generated by the extended steady state generator

$$
\left.\begin{array}{rl}
\dot{\tau} & =1 \\
\dot{w}_{e} & =S_{e} w_{e} \\
\tau^{+} & =0 \\
w_{e}^{+} & =J_{e} w_{e}
\end{array}\right\}\left(\tau, w_{e}\right) \in\left[0, \tau_{\max }\right] \times \mathbb{R}^{8+2 N},
$$

where

$$
S_{e}=\left(\begin{array}{cc}
S_{e 1} & 0_{8 \times 2 N} \\
0_{2 N \times 8} & 0_{2 N \times 2 N}
\end{array}\right), J_{e}=\left(\begin{array}{cc}
J_{e 11} & J_{e 12} \\
0_{2 N \times 8} & J_{e 22}
\end{array}\right),
$$

$R_{e}=\left(\begin{array}{cc}R_{e 1} & 0_{1 \times 2 N}\end{array}\right)$, in which $S_{e 1}=\operatorname{blkdiag}\left(S_{1}, S_{1}\right)$, $J_{e i j}=\operatorname{blkdiag}\left(J_{i j}, J_{i j}\right), i, j=1,2, R_{e 1}=\left(\begin{array}{cc}R^{\prime} & R^{\prime \prime}\end{array}\right)$ with $R^{\prime}=\left(\begin{array}{cccc}1 & 0 & 0 & 0\end{array}\right)$ and $R^{\prime}=\left(\begin{array}{llll}0 & 1 & 0 & 0\end{array}\right)$. As a matter of fact, any output signal of (15) generated by an initial condition $w(0)$ can be generated by the output of (18) with the initial condition

$$
w_{e}(0)=\left(\begin{array}{cc}
-\frac{A_{22}}{b} I_{4} & 0_{4 \times N} \\
\frac{1}{b} I_{4} & 0_{4 \times N} \\
0_{N \times 4} & -\frac{A_{22}}{b} I_{N} \\
0_{N \times 4} & \frac{1}{b} I_{N}
\end{array}\right) w(0) .
$$

We say that systems (15) and (18) are state-output equivalent. Robust regulators can be thus obtained by embedding an internal model of the extended steady state generator (18), where the dynamics $\left(S_{e}, J_{e}, R_{e}\right)$ are not affected by the uncertainties. The design procedure presented in Proposition 3 , however, does not apply "off the shelf" if the steady state generator (15) is replaced by the extended one (18). The main difference lies in the fact that while the pair $\left(S_{1}, R_{1}\right)$ is observable, the pair $\left(S_{e 1}, R_{e 1}\right)$ is clearly not. The main property that allows one to recover the design procedure of Proposition 3 in the robust framework is the fact that the unobservability subspace of the pair $\left(S_{e 1}, R_{e 1}\right)$ is invariant under $J_{e 1}$. This is formalized in the next lemma whose proof is omitted because it follows by standard arguments.

Lemma 1: Let $\mathcal{N}\left(S_{e 1}, R_{e 1}\right)$ be the unobservability subspace of the pair $\left(S_{e 1}, R_{e 1}\right)$. Then $J_{e 1} \mathcal{N}\left(S_{e 1}, R_{e 1}\right) \subseteq$ $\mathcal{N}\left(S_{e 1}, R_{e 1}\right) . \triangleleft$

The previous lemma guarantees that if $T \in \mathbb{R}^{8 \times 8}$ is any nonsingular matrix such that

$$
T S_{e 1} T^{-1}=\left(\begin{array}{cc}
S_{e 1 o} & 0_{4 \times 4} \\
\star & \star
\end{array}\right), R_{e 1} T^{-1}=\left(\begin{array}{cc}
R_{e 1 o} & 0
\end{array}\right)
$$

with the pair $\left(S_{e 1 o}, R_{e 1 o}\right) \in \mathbb{R}^{4 \times 4} \times \mathbb{R}^{1 \times 4}$ being observable, then $T J_{e 11} T^{-1}$ and $T J_{e 12}$ can be expressed as

$$
T J_{e 11} T^{-1}=\left(\begin{array}{cc}
J_{e} 11 o & 0_{4 \times 4} \\
\star & \star
\end{array}\right), T J_{e 12}=\left(\begin{array}{c}
J_{e} 12 o \\
\star
\end{array}\right) \text {, }
$$

where $\star$ are the appropriate matrices. Now, let $\left(J_{e 22 o}, J_{e 12 o o}\right) \in \mathbb{R}^{N \times N} \times \mathbb{R}^{1 \times N}$ be the observable part of the pair $\left(J_{e 22}, J_{e 12 o}\right)$. With all this in mind, it is possible to conclude that the hybrid system,

$$
\left.\begin{array}{rl}
\dot{\tau} & =1 \\
\dot{w}_{e 1} & =S_{e 1 o} w_{e 1} \\
\dot{w}_{e 2} & =0 \\
\tau^{+} & =0 \\
w_{e 1}^{+} & =J_{e 11 o} w_{e 1}+J_{e 12 o o} w_{e 2} \\
w_{e 2}^{+} & =J_{e 22 o} w_{e 2}
\end{array}\right\}\left(\tau, w_{e}\right) \in\left[0, \tau_{\max }\right] \times \mathbb{R}^{4+N},
$$

is state-output equivalent to system (18) and, in turn, to system (15). In view of this, the robust internal model can be 
designed as in (17) with $S_{1}, J_{11}, J_{12}, J_{22}$ and $R_{1}$ replaced, respectively, by $S_{e 1 o}, J_{e 11 o}, J_{e 12 o o}, J_{e 22 o}, R_{e 1 o}$.

\section{Simulation REsults}

In this section we provide two examples, the first is regarding the $\tau$-dependent internal model solution from Section III while the second is regarding the $\tau$-independent internal model solution from Section IV.

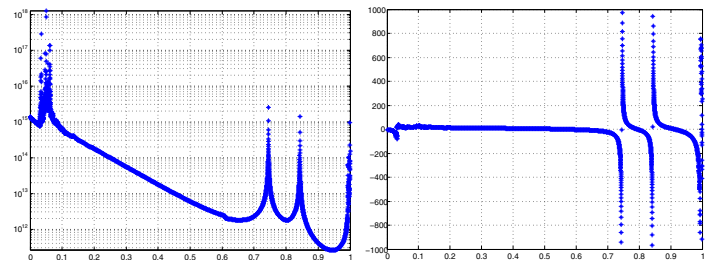

Fig. 1. Ratio of largest to smallest singular value of $\Pi_{\eta}(\tau) ; R(\tau) \Pi_{\eta}(\tau)^{\dagger}$

\section{A. $\tau$-dependent internal model, rank issue}

In this example, we provide a numerical argument that the spline generating exosystem leads to a function $\Pi_{\eta}(\tau)$ with non-constant rank over $\tau \in\left[0, \tau_{\max }\right]$. In doing so we choose the required regulator parameters as described in (9) with Proposition 1 and Proposition 2 in mind.

For the purposes of simulation we use the plant (5) defined by the parameters $b=1, A_{11}=A_{12}=0, A_{21}=1, A_{22}=1$.

We also choose the regulator parameters $\Sigma_{\mathrm{im}}=I_{8}$,

$$
F=\left[\begin{array}{ccc}
f_{1} & \ldots & f_{8} \\
I_{7} & 0_{7 \times 1}
\end{array}\right], \quad G=\left[\begin{array}{c}
1 \\
0_{7 \times 1}
\end{array}\right],
$$

where $\left(f_{1}, \ldots, f_{8}\right)$ are the coefficients such that the eigenvalues of $F=-(1,1,2,3,5,5,6,10)$.

Furthermore, the waypoints of the exosystem are chosen as $(-1,1,-2,1)$, these fully define the exosystem parameters.

Recall that $R(\tau)$ is determined by (7) using the parameters already specified. These choices lead to a solution $\Pi_{\eta}(\tau)$ to (13), that has a non-constant rank, as shown by the ratio of the maximum singular value of $\Pi_{\eta}(\tau)$ to the minimum singular value of $\Pi_{\eta}(\tau)$. This ratio is depicted in Figure 1 . Figure 1 also shows discontinuities in $R(\tau) \Pi_{\eta}(\tau)^{\dagger}$. Since $R(\tau) \Pi_{\eta}(\tau)^{\dagger}$ is not a scalar function we plot one element of it to show its discontinuity.

This result precludes the use of the $\tau$-dependent regulator from Section III, so a new method for designing the internal model is needed. The results from Section IV fulfill this need.

\section{B. $\tau$-independent internal model}
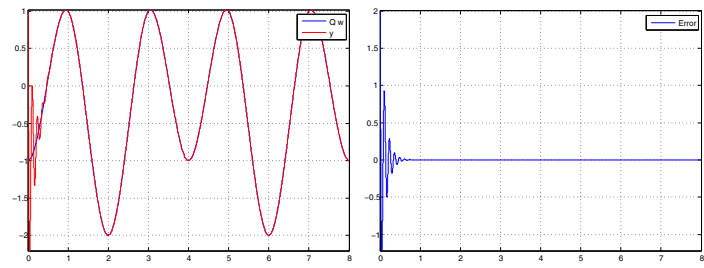

Fig. 2. $Q w, y$; Error, $e=y-Q w$
Here we present an example using the ideas from Section IV to build an internal model for the spline generating exosystem. We use the same plant parameters, $A_{11}, A_{12}$, $A_{21}, A_{22}$ and $b$, as in the previous example. We also use the same exosystem waypoints. Consequently, the only change is the choice of regulator parameters.

Here we choose the regulator as in Proposition 3, with $R$ as in (14) and $\Gamma_{\mathrm{im}}=R$. We then choose $K_{1}$ and $K_{2}$ by placing the poles of $\left(J_{22}+K_{2} L_{2}\right) \in \mathcal{D}_{1}$ and by placing $\operatorname{Re}\left[\operatorname{eig}\left(S_{1}+K_{1} R_{1}\right)\right] \leq g$. Here we pick eig $\left(J_{22}+K_{2} L_{2}\right)=$ $(0.1127,0.1815,0.1906,0.1913)$ and $\operatorname{eig}\left(S_{1}+K_{1} R_{1}\right)=$ $-(15,16.5,18,19.5)$. Lastly, we choose $\kappa=50$.

As can be seen in Figure 2, the problem of hybrid output regulation has been solved for this system. Figure 2 shows the spline and error tracking.

\section{CONCLUSIONS}

The paper focused on the problem of robust tracking of cyclic reference signals obtained by spline interpolation for linear systems. The spline-based signal has been modeled as an output of a hybrid exosystem and a hybrid internal model-based regulator has been designed that is able to steer the tracking error to zero despite possible parametric uncertainties. Future works on the subject aim at formulating the problem in a nonlinear hybrid setting.

\section{REFERENCES}

[1] N. Cox, A. R. Teel, L. Marconi, "Hybrid Output Regulation for Minimum Phase Linear Systems", Proceedings of the 2011 IEEE American Control Conference, San Francisco, 2011.

[2] N. Cox, L. Marconi and A. R. Teel , "Hybrid Output Regulation with Unmeasured Clock", Proceedings of the 50th IEEE Conference on Decision and Control, 2011.

[3] E.J. Davison, "The robust control of a servomechanism problem for linear time-invariant multivariable systems", IEEE Trans. Autom. Contr., AC-21: 25-34, 1976.

[4] A. De Luca, L. Lanari, G. Oriolo, "A sensitivity approach to optimal spline robot trajectories", Automatica, Vol. 27, N. 3, 1991.

[5] B.A. Francis, "The linear multivariable regulator problem", SIAM J. Contr. Optimiz., 14: 486-505, 1977.

[6] R. Goebel, R. Sanfelice, A. R. Teel, "Hybrid dynamical systems", IEEE Control System Magazine, pp. 28-93, April 2009.

[7] T. M. Howard, A. Kelly, "Trajectory and Spline Generation for All-Wheel Steering Mobile Robots", Proceedings of the the 2006 IEEE/RSJ International Conference on Intelligent Robots and Systems (IROS '06), 2006.

[8] A. Isidori and C.I. Byrnes. "Output regulation of nonlinear systems", IEEE Trans. Autom. Contr., AC-25: 131-140, 1990.

[9] L. Marconi and A.R. Teel, "Hybrid Linear Regulation", Submitted to IEEE Transaction on Automatic Control, 2012.

[10] L. Marconi, A. R. Teel, "A Note About Hybrid Linear Regulation", 49th IEEE Conference on Decision and Control, Atlanta, USA, 2010.

[11] L. Schumaker, "Spline Functions: Basic Theory", Cambridge Mathematical Library 3rd Edition, 2007.

[12] B. Siciliano, L. Sciavicco, L. Villani, "Robotics: Modeling, Planning and Control", (Advanced Textbooks in Control and Signal Processing), Springer Verlag,

[13] L. Sonneveldt, E. R. van Oort, Q.P. Chu, J.A. Mulder, "Nonlinear adaptive flight control law design and handling qualities evaluation" Proceedings of the 48th IEEE Conference on Decision and Control, 2009.

[14] G.W. Stewart, "On the continuity of the generalized inverse", SIAM J. Appl. Math., Vol. 17, No. 1, pp. 33-45, Jan 1969.

[15] A. R. Teel, L. Marconi, "Stabilization for a class of minimum phase hybrid systems under an average dwell-time constraint", to appear on International Journal of Robust and Nonlinear Control, March 2010. 